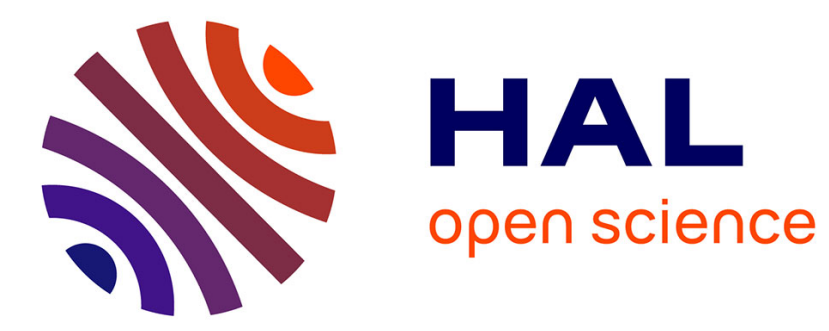

\title{
Les fouilles exécutées à Alésia en 1942 par la Société des Sciences historiques et naturelles de Semur (Côte-d'Or)
} Jules Toutain

\section{To cite this version:}

Jules Toutain. Les fouilles exécutées à Alésia en 1942 par la Société des Sciences historiques et naturelles de Semur (Côte-d'Or). Gallia - Fouilles et monuments archéologiques en France métropolitaine, 1943, 1 (2), pp.133 158. 10.3406/galia.1943.1972 . hal-01920346

\section{HAL Id: hal-01920346 \\ https://hal.science/hal-01920346}

Submitted on 25 Feb 2020

HAL is a multi-disciplinary open access archive for the deposit and dissemination of scientific research documents, whether they are published or not. The documents may come from teaching and research institutions in France or abroad, or from public or private research centers.
L'archive ouverte pluridisciplinaire HAL, est destinée au dépôt et à la diffusion de documents scientifiques de niveau recherche, publiés ou non, émanant des établissements d'enseignement et de recherche français ou étrangers, des laboratoires publics ou privés.

\section{(ㅇ)(1) $\$$}

Distributed under a Creative Commons Attribution - NonCommercial - NoDerivatives| 4.0 


\title{
LES FOUILLES EXÉCUTEES A ALESIA EN 1942
}

\author{
par la \\ Société des Sciences historiques el naturelles de Semur (Côte-d'Or) \\ Rapport par M. J. Toutain
}

\section{I. - L'édifice à double colonnade.}

Pendant les mois d'août et de septembre, nos efforts ont surtout visé à déblayer en profondeur l'édifice à double colonnade, dont le dégagement entrepris en 1908 par la Société des Sciences de Semur avait dû être abandonné à la suite de difficultés techniques. Quand nous avons rencontré un pavement antique, nous l'avons respecté; partout ailleurs nous nous sommes arrêtés seulement quand nous avons atteint le sol naturel du Mont Auxois; parfois même nous avons fouillé plus bas lorsque les murs descendaient au-dessous de ce niveau, jusqu'aux dernières assises de ces murs. Cette exploration profonde nous a permis de compléter, de préciser, quelquefois même de corriger, les observations, à peu près exclusivement superficielles, qui avaient été faites en 1908 et 1909. Toute la partie méridionale de l'édifice est aujourd'hui découverte et peut être décrite sans lacune, ni crainte d'erreurs. Ce n'est pas à dire que l'interprétation en soit absolument certaine et ne se heurte pas à quelques difficultés; mais les vestiges en sont complètement dégagés et se prêtent̀ à une étude devenue facile.

La façade, tournée vers le sud, est marquée par une double colonnade, s'allongeant de l'ouest à l'est sur 34 mètres de développement (fig. 1). L'existence de cette double colonnade est attestée par deux rangées exactement parallèles de socles carrés en pierre (fig. 2). Les socles de la colonnade extérieure mesurent 1 mètre de côté; ceux de la colonnade intérieure, $0 \mathrm{~m}$. 65. La distance d'un socle à l'autre, sur chaque rangée, est $2 \mathrm{~m}$. 50 à $2 \mathrm{~m}$. 60 . Les deux rangées de socles reposent sur deux murs continus, épais l'un de $0 \mathrm{~m}$. 45, l'autre de $0 \mathrm{~m} .55$ à $0 \mathrm{~m}$. 60. A la surface de quelques-uns de ces socles se voient encore les traces des colonnes qu'ils portaient; un tronçon de fût de colonne, trouvé en place en 1908 , mesure $0 \mathrm{~m} .58$ de diamètre. On estime que, dans l'ordre toscan, la 


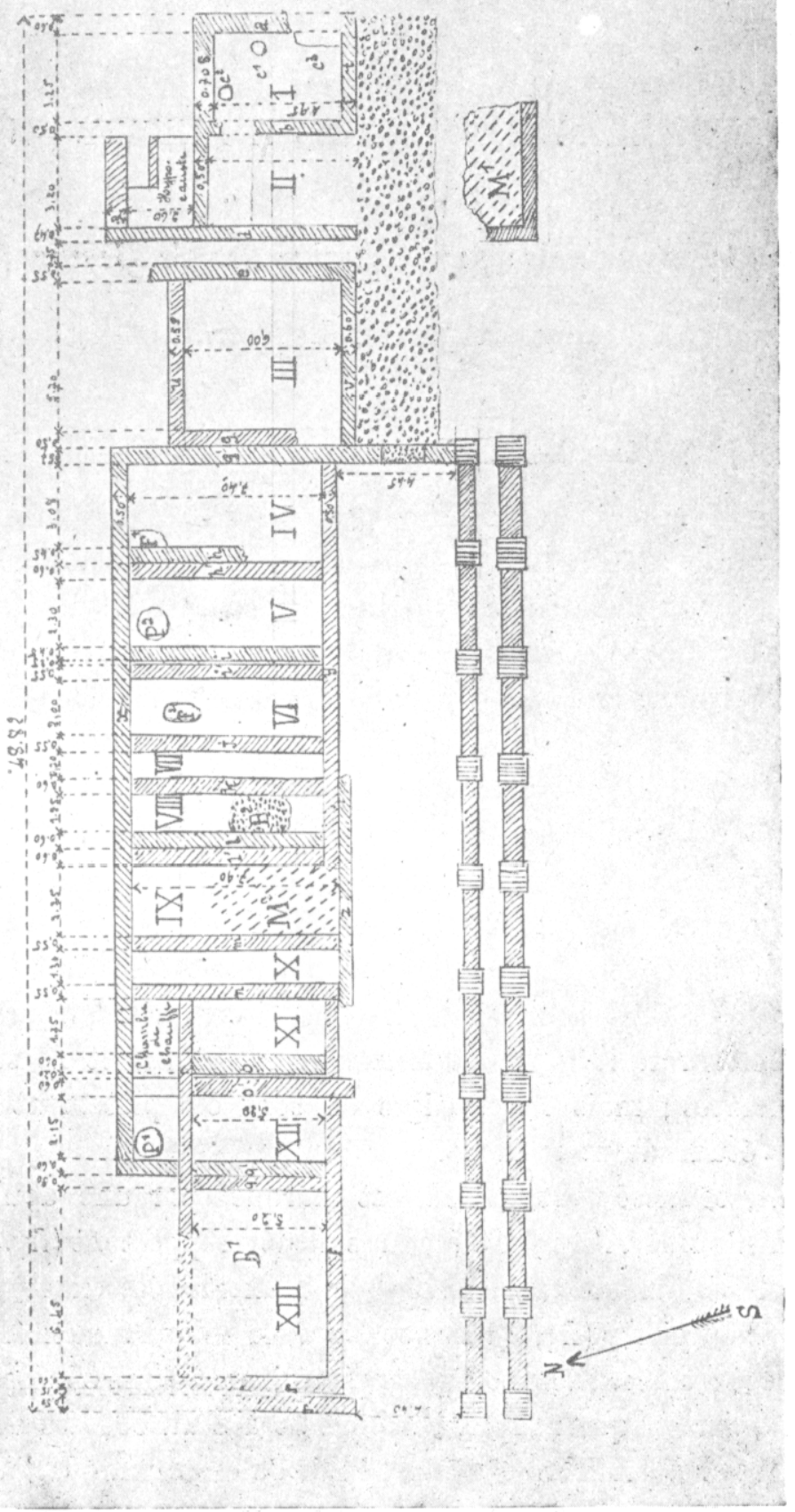




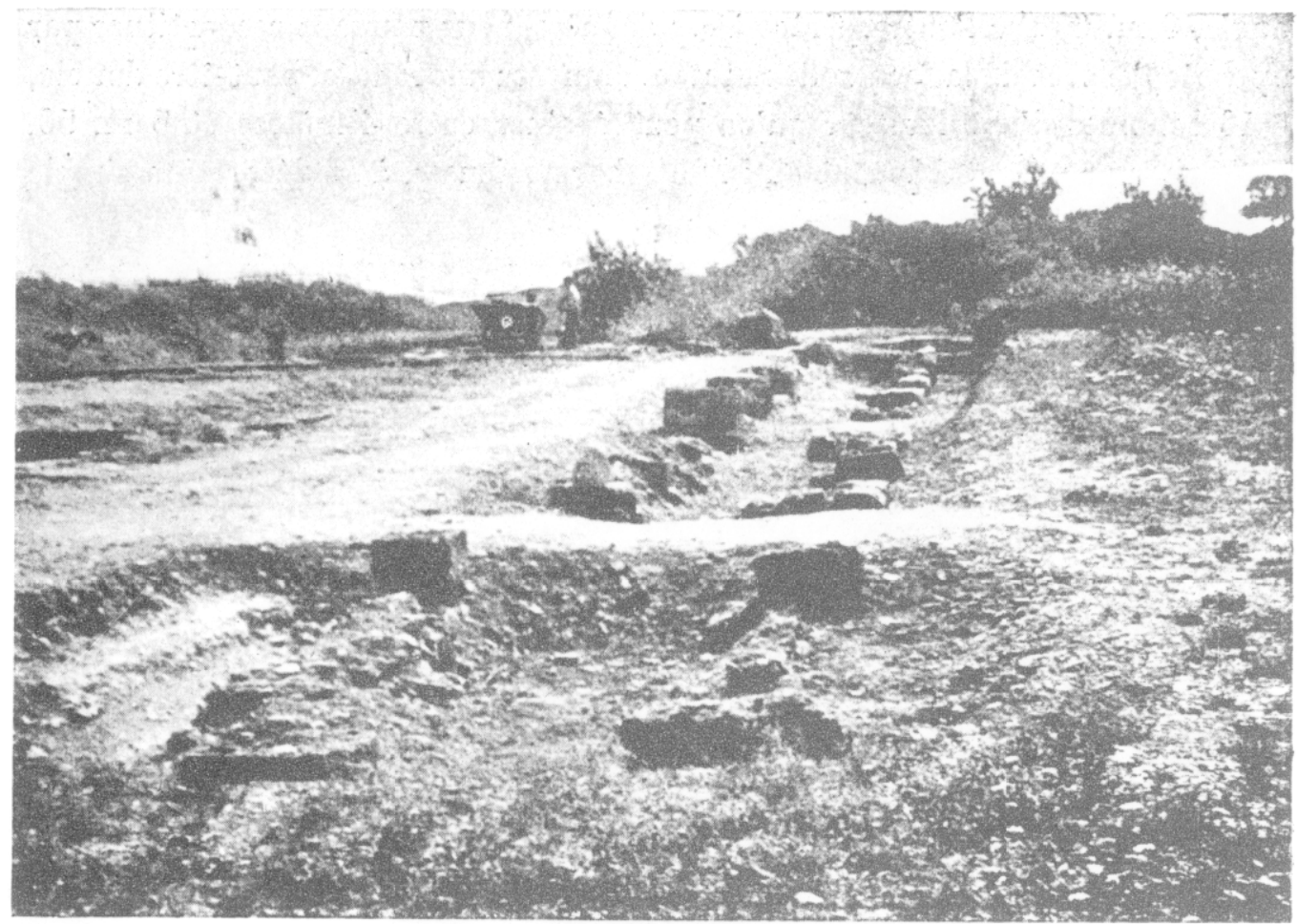

Fig. 2. La double colonnade, vue de l'ouest.

hauteur de la colonne a varié de 7 diamètręs à près de 10 ; dans ces conditions la colonnade aurait été haute de 4 mètres à $5 \mathrm{~m}$. 50 . Elle était couronnée d'un entablement dont nous avons retrouvé un angle (fig. 3) : la décoration en est très sobre ; entre l'architrave et la corniche, toutes deux représentées par des moulures au profil classique, la frise est rëmplacée par une ligne de denticules, dont les dimensions $(0 \mathrm{~m} .08 \times 0 \mathrm{~m} .07$ sur 0 m. 09 de hauteur) s'accordent avec celles des moulures. Le morceau de cet entablement que nous possédons mesure $0 \mathrm{~m}$. 78 sur un côté de

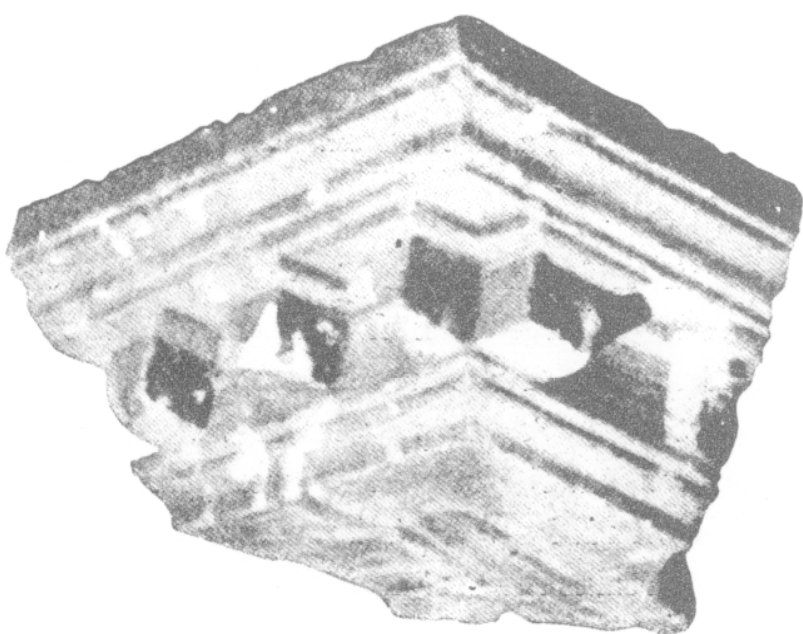

FIG. 3. Angle d'entablement. 
l'angle, $0 \mathrm{~m} .60$ sur l'autre. Grâce à ces données, nous pouvons reconstituer par la pensée l'aspect de la façade de notre monument: une rangćc, pcut-ĉtre double, de 10 colonnes d'une hauteur qu'on peut évaluer entre 4 mètres et $5 \mathrm{~m}$. 50 , couronnée par un entablement aux lignes harmonieuses, à la décoration simple et bien proportionnée.

Nous disons : une rangée peut-être double, parce que la question se pose

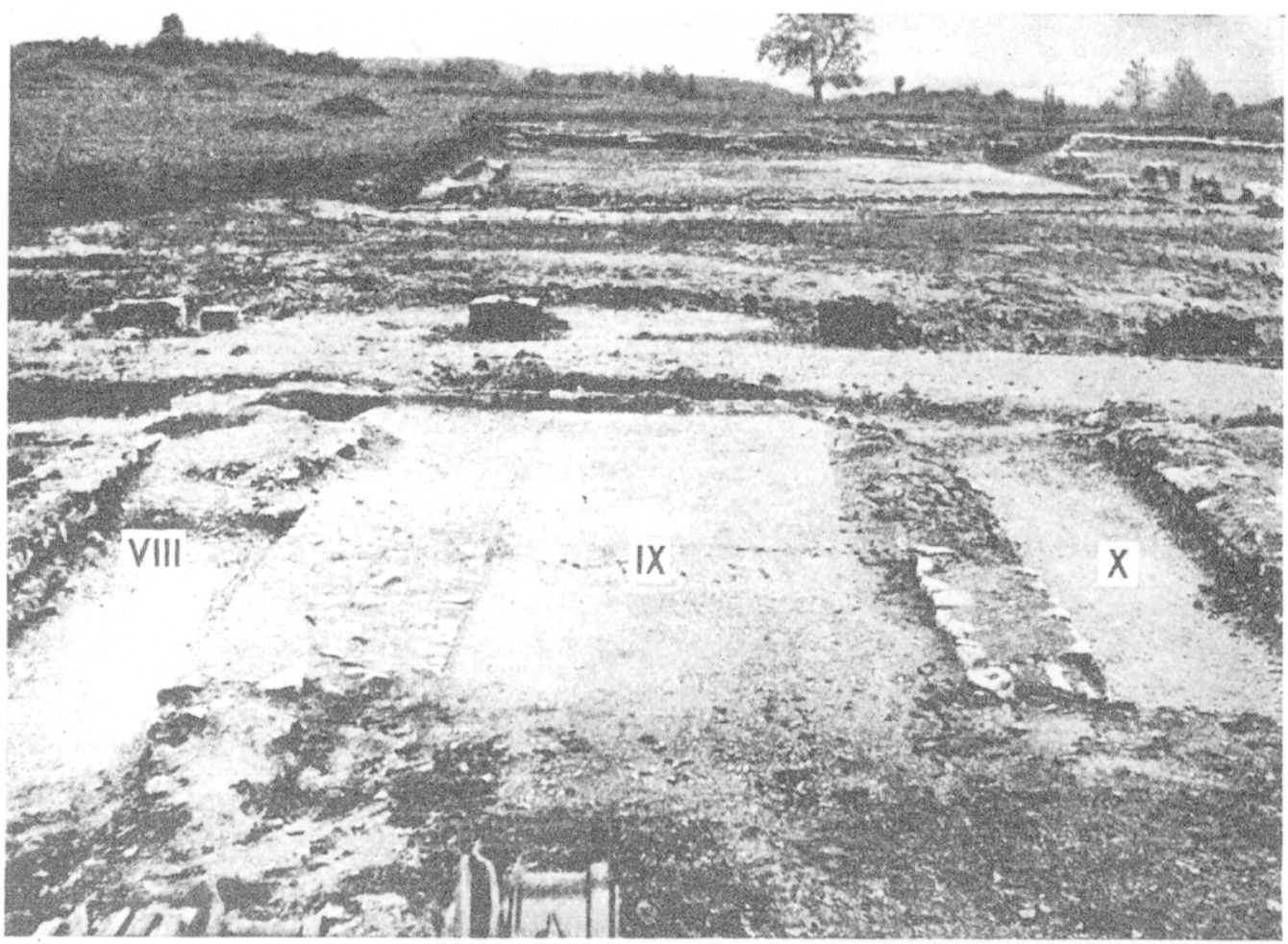

Fig. 4. Partie centrale de l'édifice : chambres VIII, IX et X.

de savoir si les deux rangées de colonnes existaient ensemble ou si elles se sont succédé. Les deux files de socles sont très proches l'une de l'autre; et l'on ne comprend pas très bien pourquoi cette colonnade de façade a été ainsi géminée. Mais d'autre part les socles des deux files sont placés en face les uns des autres, aussi exactement que possible. Il semble que la construction de l'édifice ait subi des remaniements, comme nous aurons l'occasion de le signaler d'après certains indices. En résumé, les découvertes faites sur place ne fournissent aucune certitude; elles ne nous permettent pas de conclure dans un sens plutôt que dans l'autre. 
La façade de l'édifice donne sur la voie romaine, qui passe au nord du théâtre et de la basilique civile; du centre de la façade, entre les colonnes 5 et 6 de chaque rangée, un pavé en hérisson large de $2 \mathrm{~m}$. 60 joint l'édifice à la voie et nous indique que l'entrée se trouvait là.

Après avoir franchi la colonnade, on se trouvait dans une vaste galerie ou dans un portique, qui occupait toute la longueur du monument sur une profondeur de $4 \mathrm{~m} .50$ environ. Aucun mur transversal n'a été aperçu dans cet espace. Le pavement de cette galerie consistait en un béton de couleur blanchâtre ; un premier béton fut établi sur des décombres, provenant sans doute d'une construction antérieure; à $0 \mathrm{~m}$. 20 au-dessus un second béton de même nature et de même couleur révèle une réparation ou un remaniement.

C'est au delà de cette galerie ou de ce portique que se dessine le plan général de l'édifice. Deux murs partant des deux extrémités de la colonnade de façade l'encadrent à l'est et à l'ouest. Parallèlement à la colonnade, la galerie ou le portique se trouvait limité de bout en bout par un mur dans lequel devaient s'ouvrir des portes ou des baies donnant accès à une série de pièces toutes perpendiculaires à la direction de la façade. La pièce centrale (IX du plan fig. 1), longue de $7 \mathrm{~m}$. 90, large de $2 \mathrm{~m}$. 25, parait avoir servi d'entrée, de vestibule (fig. 4); elle est pavée d'une mosaïque en petits cubes de terre cuite, à peu près intacte encore aujourd'hui dans sa plus grande partie. A droite et à gauche se succèdent diverses pièces ; à droite, c'est-à-dire à l'est, elles ont toutes la même longueur du nord au sud, soit $7 \mathrm{~m} .40$ dans œuvre ; mais elles sont de largeur très inégale, allant en croissant d'ouest en est : la pièce VIII du plan est large de $1 \mathrm{~m} .25$; le $\mathrm{n}^{0}$ VII, large seulement de $0 \mathrm{~m}$. 70 parait n'avoir été que le passage peut-être d'une conduite d'eau ; VI est large de 2 mètres ; V de $2 \mathrm{~m}$. 30 ; IV de $3 \mathrm{~m} .08$ (fig. 5 ). Ces diverses pièces sont séparées les, unes des autres par des murs dont l'épaisseur varie de $0 \mathrm{~m}$. 55 à $0 \mathrm{~m}$. 60 et qui sont parfois doubles, par exemple entre IX et VIII, entre VI et V, entre V et IV. En VIII subsiste un fragment de béton $\left(\mathrm{B}^{2}\right)$ composé de petits cailloux noyés dans un mortier très résistant, en VI et en IV ont été retrouvés deux emplacements de foyers $\left(\mathrm{F}^{2}\right)\left(\mathrm{F}^{1}\right)$; en $\mathrm{V}$ a été découvert un puisard $\left(\mathrm{P}^{2}\right)$ profond de $2 \mathrm{~m} .80$ où nous avons recueilli beaucoup de morceaux de poteries dont nous parlerons plus loin. Les fondations du mur, qui limite ces pièces au sud et les sépare de la grande galerie, descendent parfois plus bas que le sol naturel; il semble que nous trouvions là des vestiges d'une construction plus ancienne.

A gauche, c'est-à-dire à l'ouest de la pièce centrale, le plan de l'édifice est un peu différent. Si la pièce $X$, très étroite (elle n'a que $1 \mathrm{~m}$. 30 de large) a la même longueur que les pièces situées à l'est, soit $7 \mathrm{~m}$. 40 dans œuvre, au delà les pièces XI et XII sont réduites en longueur à $5 \mathrm{~m}$. 20, et deux pièces, beaucoup 


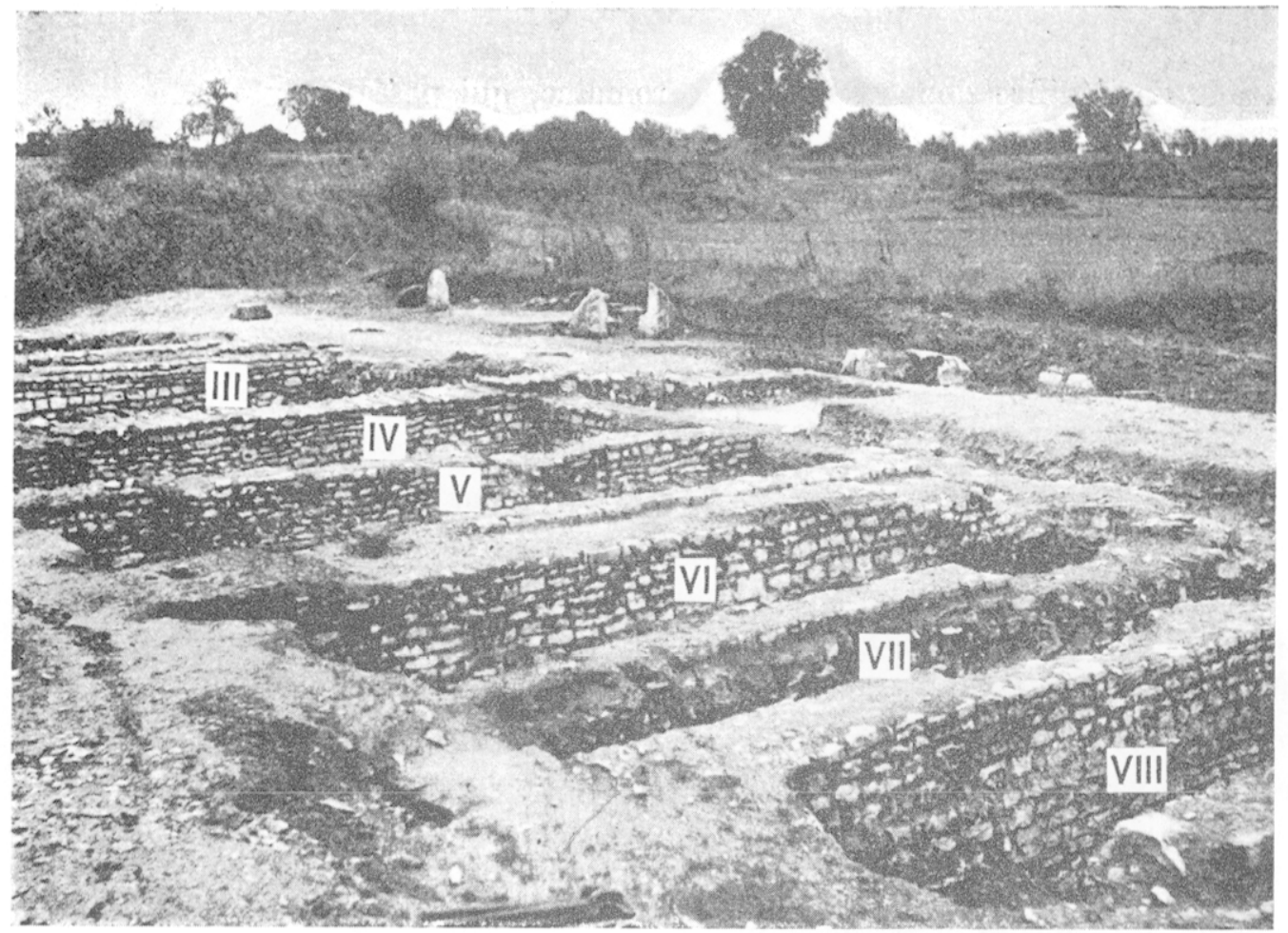

Fig. 5. Partie est de l'édifice : chambres III IV, V, VI, VII, VIII.

Fig. 6. Partie ouest de l'édifice : chambres XI, XII,

XIII. Au premier plan, l'hypocauste.

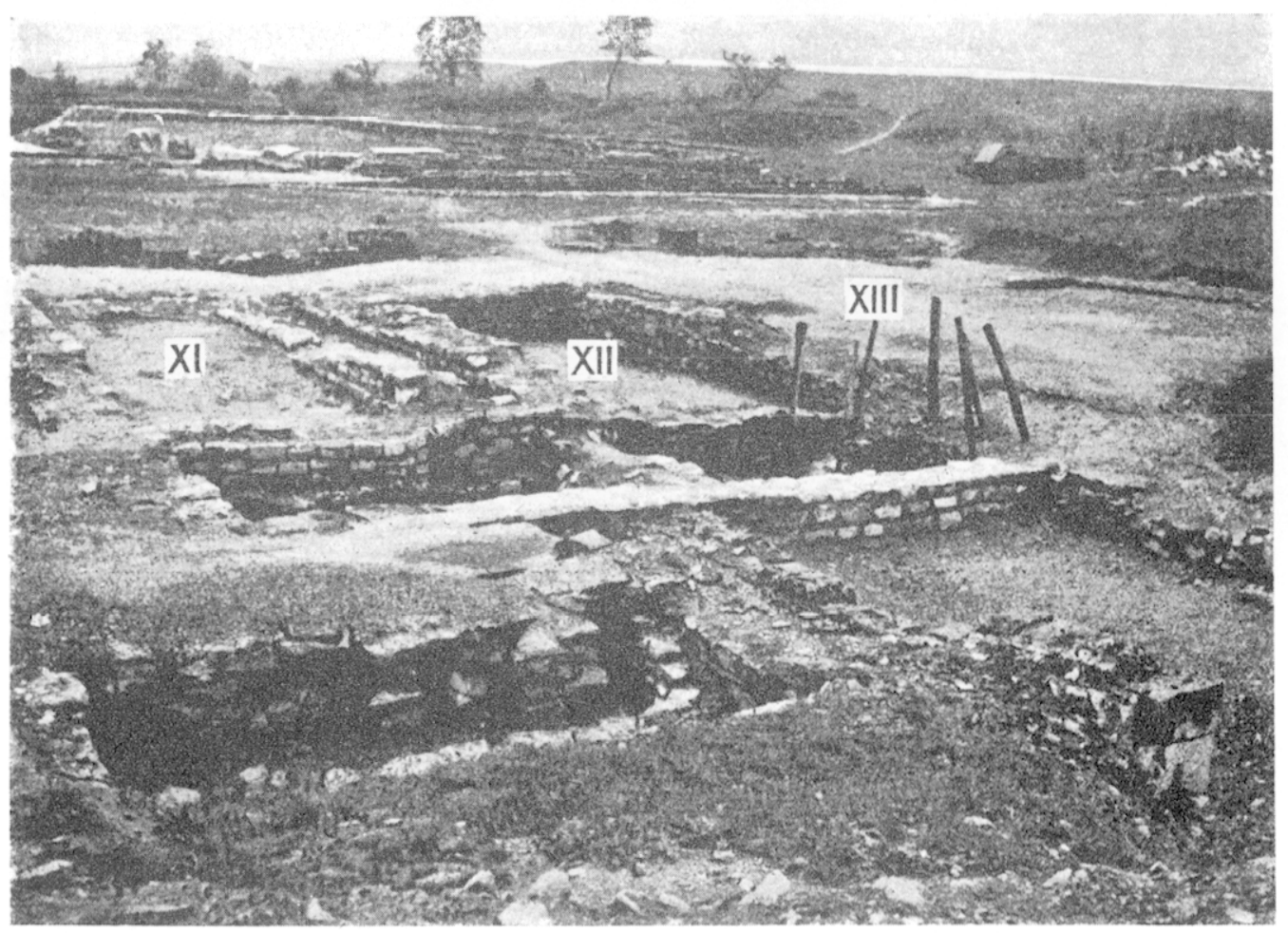


plus petites, à peu près carrées, mesurant l'une $1 \mathrm{~m}$. 75 de côté, l'autre $2 \mathrm{~m}$. 15 , en sont séparées par un mur transversal (fig. 6). Sous le sol de ces deux petites pièces existe un hypocauste que nous décrirons plus loin; dans l'angle nord-ouest de l'une d'entre elles, la plus occidentale, se trouve un puisard $\left(\mathrm{P}^{1}\right)$, moins large que celui de la pièce V. Les deux pièces XI et XII sont séparées par deux murs accolés étroitement l'un à l'autre ; elles sont larges, la pièce XI de $1 \mathrm{~m}$. 75, la pièce XII de $2 \mathrm{~m}$. 15. Au delà de la pièce XII, se trouvait une vaste pièce rectangulaire mesurant $5 \mathrm{~m}$. 20 du nord au sud, comme les pièces XI et XII, et $6 \mathrm{~m}$. 45 de l'est à l'ouest. Elle était pavée d'un béton de chaux assez grossier $\left(B^{1}\right)$. Sauf cette dernière pièce, toutes les autres sont limitées à leur extrémité nord par un mur continu épais de $0 \mathrm{~m}$. 50, qui rejoint le mur latéral de l'est, mais dont nous n'avons pas retrouvé trace à l'ouest au delà de la pièce XII.

Tel est le plan général de la partie du monument que nous avons explorée tant en profondeur qu'en surface. Nous devons ajouter qu'à l'extrémité orientale nous avons dégagé, en dehors du mur latéral de l'est, une pièce à peu près carrée : 6 mètres $\times 5 \mathrm{~m}$. 70, dont nous ne savons pas si c'était une annexe du monument ou une construction indépendante, la pièce III. Elle est nettement séparée de l'habitation que nous avons fouillée à l'extrémité est de notre chantier (pièces II et I) par un espace libre large de 0 m. 75 ; au contraire son mur ouest est directement contigu au mur latéral de l'édifice à double colonnade (fig. 7). En outre, par sa situation et par ses dimensions, elle parait symétrique de la pièce XIII qui occupe l'angle sud-ouest.

Un dernier détail doit être mentionné dans cette description du monument découvert : à l'extrémité orientale de la galerie qui s'allonge derrière la colonnade, le mur latéral de l'est semble interrompu par un seuil large d'environ 2 mètres. Là devait se trouver une entrée secondaire, à laquelle aboutissait une rue dont il sera question ci-dessous.

Il convient maintenant d'insister sur quelques points particuliers et sur plusieurs découvertes spéciales.

L'un des éléments caractéristiques de l'édifice est l'hypocauste qui en occupe. une partie et qui servait à chauffer tout un ensemble de pièces. Il avait été déjà aperçu lors des fouilles de 1908. Lorsqu'on le dégagea, le foyer, établi sur un pavé en hérisson, portait encore une couche épaisse de cendres et de charbon. Dans les décombres on recueillit des débris de mosaïques en terre cuite, provenant des pavements des pièces chauffées et des fragments de boisseaux en terre cuite striés à l'extérieur, restes de la tubulation qui permettait à l'air chaud de circuler le long des murs. Nous avons nous-mêmes retrouvé, dans la petite pièce située au nord de la pièce XI, une partie de l'hypocauste, en même temps que les piliers qui soutiennent les pavements des pièces $\mathrm{XI}$ et $\mathrm{X}$. Il est probable, mais 
nous n'avons pas pu le vérifier, que la pièce IX se trouvait également chauffée. Jusqu'où l'hypocauste s'ćtendait-il ? Le souci de ne détruire aucun vestige antique nous a empêchés de nous en rendre compte exactement.

Dans la partie de notre fouille située à l'est de la double colonnade, nous avons trouvé, en fort mauvais état, mais pourtant reconnaissables sans aucun

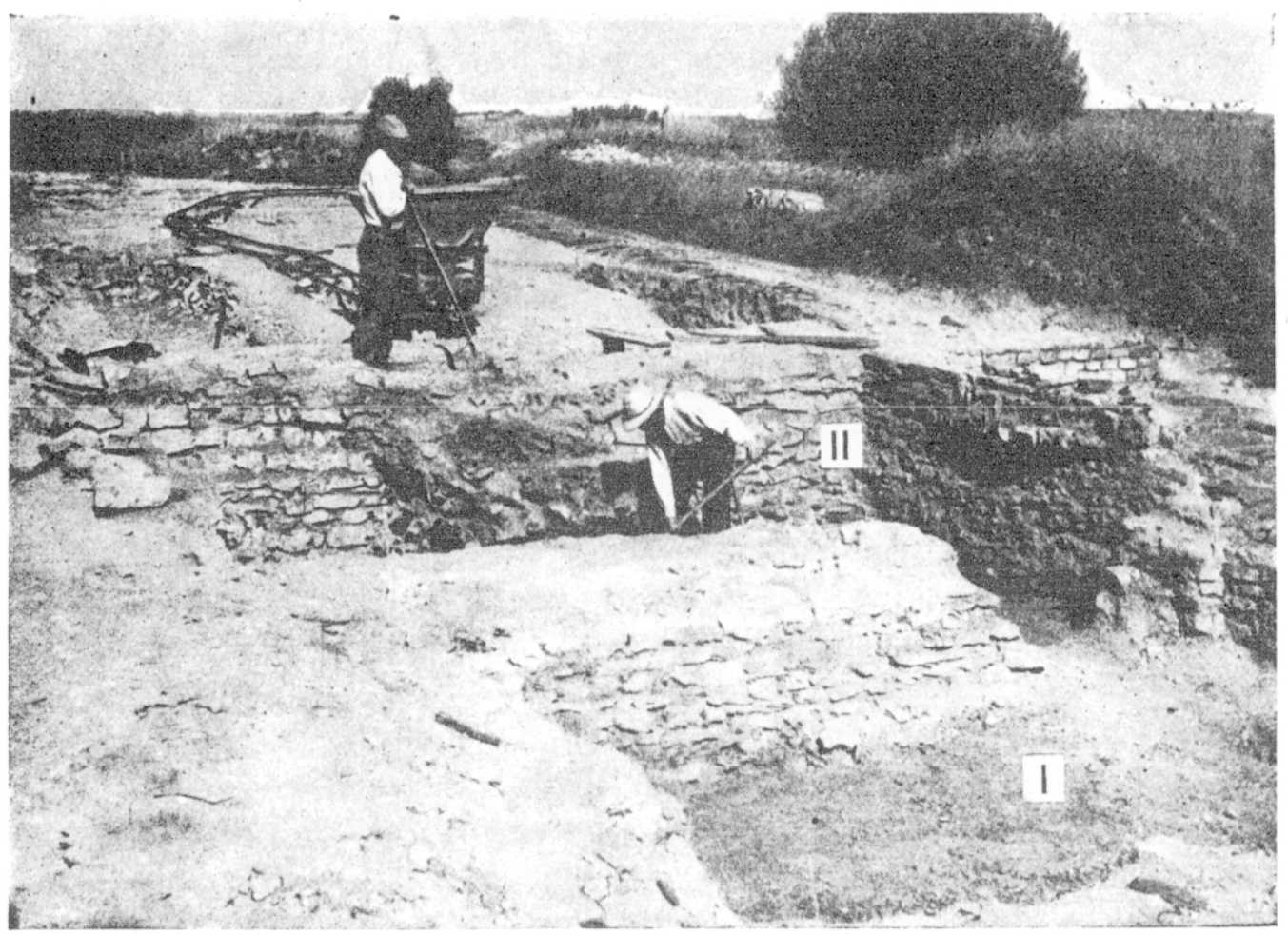

Fic. 7. L'habitation de l'est : chambres I et II.

doute, les parties inférieures de deux demi-colonnes en pierre avec leurs socles rectangulaires. Le diamètre des deux demi-colonnes, autant qu'on peut le mesurer, est de $0 \mathrm{~m} .50$, la plus grande hauteur conservée n'est que de $0 \mathrm{~m} .45$, les socles mesurent $0 \mathrm{~m} .59 \times 0 \mathrm{~m} .25$ avec une hauteur de $0 \mathrm{~m}$. 33. En raison de leur forme même, ces demi-colonnes devaient être adossées à quelque mur : ne peut-on pas supposer qu'elles encadraient l'entrée secondaire de l'édifice, à l'extrémité orientale de la grande galerie ? Le diamètre de ces demi-colonnes est très voisin de celui du fût trouvé lors des premières fouilles et qui appartenait probablement à l'une des colonnes de la façade.

Des matériaux employés dans la construction de l'édifice, nous avons recueilli 
quelques débris : 5 pierres de forme rectangulaire dont une des faces est ornée de traits disposés en arêtes de poisson, analogues aux pierres du Musée et de la région de Sens signalées par Mlle Augusta Hure $(1) ;-2$ morceaux de couvrejoints en pierre, dans la face concave desquels un trou a été ménagé pour permettre sans doute de les fixer solidement à la charpente sous-jacente ; 一 un certain nombre de morceaux de pierres tendres sciées; la découverte dans le même chantier de fouilles de ces pierres tendres sciées et des morceaux de couvre-joints en pierre nous révèle que la toiture du monument était conforme au système que M. l'architecte Chaussemiche a reconstitué en 1909 et qui est ainsi décrit dans notre rapport sur les fouilles exécutées en 1909 par la Société des Sciences historiques et naturelles de Semur (2) : "Cette toiture était faite de pierres tendres sciées; elle s'est effondrée sur place; les pierres ont été retrouvées au fond de la pièce ainsi couverte à peu près dans la position qu'elles devaient occuper quand elles en constituaient le toit... Des pierres de forme demi-cylindrique placées bout à bout jouaient le rôle de tuiles faitières ; de l'un et l'autre côté de cette rangée des pierres plates rectangulaires légèrement imbriquées constituaient le double versant. "Le détail nouveau, qui ressort de notre découverte, c'est la présence, dans la rainure demi-cylindrique, du trou destiné probablement à assurer la fixité du couvre-joint; ce détail permet de compléter et de préciser le mode de toiture; - une pierre ronde très dure, mesurant $0 \mathrm{~m} .32$ de diamètre et $0 \mathrm{~m} .12$ de hauteur; au centre de sa surface supérieure, légèrement convexe, une petite excavation circulaire (diamètre $0 \mathrm{~m} .05$, profondeur $0 \mathrm{~m} .04$ ) a été creusée pour y fixer un anneau, et la pierre ainsi munie servait peut-être à boucher un orifice de citerne ; - une pierre ronde en granit, dont le diamètre est de $0 \mathrm{~m} .53$ et la hauteur de $0 \mathrm{~m} .13$; nous ne voyons quel pouvait en être l'usage.

Les divers murs que nous avons dégagés ne sont pas tous de la même facture ; si plusieurs d'entre eux sont construits en petits moellons carrés bien appareillés, d'autres sont faits de pierres inégales et présentent un aspect plus grossier. Des réparations ont dû être faites à certaines parties de l'édifice, suivant toute apparence à une époque assez basse. Les grandes lignes du plan ne paraissent pas avoir été changées. La fouille en profondeur, qui nous a permis de mieux lire ce plan sur le terrain, ne nous a point révélé la complication qui avait paru en 1908 caractériser ce monument, où Victor Pernet avait cru reconnaitre " un grand nombre de murs d'épaisseurs diverses; chevauchant les uns sur les autres et s'intriquant entre eux ». Il ajoutait d'ailleurs : "Qu'on ne pourra

(1) Bulletin archéologique du Comité, 1932-1933, p. 234 et suiv.

(2) Bulletin de la Société des Sciences de Semur, t. XXXVII, 1910-1911, p. 95-96, pl. IV et V. 
comprendre la disposition intérieure du monument qu'avec un plan détaillé (1). " C'est ce plan que notre campagne de 1942 nous a permis de dresser pour toute la partie méridionale de l'édifice.

Pouvons-nous maintenant déterminer avec certitude ce qu'était cet édifice ? Ce qui ne laisse place à aucun doute, c'est que, par son étendue, par sa décoration, par son plan général, il se révèle comme un monument public, sa façade de 34 mètres, sa colonnade, qu'elle ait été simple ou double, l'aspect presque classique de son entablement, la galerie ou le portique qui occupait derrière la façade toute la longueur de l'édifice ne peuvent convenir à une habitation privée. Parmi les monuments publics, qui se rencontrent dans les cités provinciales de l'Empire romain, il ne saurait être question ni d'un sanctuaire, ni d'un théâtre, ni d'une basilique, ni d'une curie. La succession des pièces parallèles qui s'ouvraient sur la galerie ou portique puurrait faire penser à une série de logettes comparables à celles qui ont été découvertes à Saint-Bertrand-de-Comminges, au voisinage du Forum, et dans lesquelles on croit reconnaitre le marché de la ville; mais ici cette interprétation ne saurait se concilier avec la présence de l'hypocauste destiné à chauffer plusieurs de ces pièces. C'est précisément cet hypocauste qui nous fournit, croyons-nous, la solution du problème. Nous sommes ici en présence des thermes d'Alésia. Certes on ne saurait les comparer aux balnéaires des grandes villes du monde romain, ni à ceux de certaines capitales de civitates gallo-romaines, ni même à ceux de maintes cités africaines telles que Thamugadi (Timgad) ou Cuicul (Djemila). Il ne faut pas oublier que, si Alésia devait à son histoire une renommée el un prestige incontestables, au point de vue administratif elle était seulement le chef-lieu d'un pagus de la cité des Éduens. Toute comparaison invoquée pour expliquer tel ou tel de ces monuments doit s'inspirer de ce fait. Or nous croyons pouvoir, en ce qui concerne le plan général de l'édifice, le rapprocher du plan des thermes du Vieil-Évreux et de celui des thermes de Badenweiler. Il y a sans doute des différences assez marquées dans l'orientation de l'ensemble et dans la forme des diverses salles. Mais au Vieil-Évreux et à Badenweiler comme à Alésia, l'édifice se trouve divisé en deux parties à peu près symétriques et l'on a voulu reconnaître ici et là un établissement thermal formé de deux parties, l'une réservée aux hommes, l'autre aux femmes (2). Les deux grandes salles, presque carrées, qui occupent les extrémités est et ouest de l'édifice d'Alésia, trouvent leurs similaires dans les deux salles rondes situées aux deux extrémités des

(1) Ibid., t. XXXVI, 1908-1909, p. 393.

(2) Pour les thermes du Vieil-Evreux, Em. Espérandieu, Les fouilles du Vieil-Éureux, dans Bulletin de la Société française des Fouilles archéologiques, t. III, p. 84, fig. 16 et suiv.; t. IV, p. 78, fig. 12. - Pour les thermes de Badenweiler, Daremberg et Saglio, Dictionnaire des Antiquités, s. v. Balneum, t. I, p. 661. 
thermes du Vieil-Évreux, dans les deux couples de salles qui occupent les extrémités est et ouest des thermes de Badenweiler. Au Vieil-Évreux et à Badenweiler comme à Alésia, les diverses salles sont disposées parallèlement les unes aux autres suivant un plan régulier.

Nous n'ignorons pas les objections qui peuvent nous être opposées. Un seul hypocauste, nous dira-t-on, paraît bien insuffisant pour chauffer ces thermes; d'autre part aucune trace de canalisation ni d'aqueduc n'a été reconnue : comment l'établissement était-il alimenté d'eau? Ces objections ne sont pas probantes.

Pour compléter la source de chaleur qu'était l'hypocauste, il arrivait qu'on disposât soit des foyers au voisinage immédiat des baignoires (alvei), soit des réchauds dans telle ou telle salle. Le premier procédé est signalé dans des bains découverts à Khamissa, en Algérie (1). La présence de réchauds ou brasiers de bronze dans les salles des thermes est attestée pour Pompéi (2). Aux thermes dits du Forum le tepidarium était chauffé à l'aide d'un réchaud de bronze, qui porte le nom du donateur M. Nigidius Vaccula; un réchaud du même genre, portant le même nom, a été trouvé dans les thermes dits de Stabies et Mau suppose qu'il fut à l'origine destiné à chauffer le lepidarium, avant que le chauffage par hypocaustes fût installé dans le balnéaire. Les traces de foyers observés dans les chambres IV et VI des thermes d'Alésia, assez éloignées de l'hypocauste, témoignent peut-être d'un chauffage, certainement plus rudimentaire et plus grossier, mais de caractère analogue.

Quant à l'alimentation en eau, si d'habitude elle était assurée par un aqueduc qui venait de quelque source parfois lointainc, il n'est pas sans exemple qu'elle eût pour origine soit un ou plusieurs puits, soit une ou plusieurs citernes. A Thuburdo Majus en Tunisie, aussi bien les thermes d'hiver que les thermes d'été étaient alimentés par un puits (3); à Thysdrus, également en Tunisie, on avait recours, pour fournir aux thermes l'eau nécessaire, à deux puits et cinq citernes, " où l'on emmagasinait l'eau de pluie tombant sur le monument et les constructions voisines " (4); à Sufetula, encore en Tunisie, l'eau qui servait à un vaste établissement thermal était recueillie dans une longue citerne située en bordure de l'édifice (5). Il en était de même en Algérie à Madaure (6); à

(1) Bulletin archéologique du Comité..., 1916, p. 204 : «... de vastes alvei dont l'un, celui de l'ouest, est flanqué d'un puissant fourneau $n$; l'alveus sud obtenait sa température élevée par un fourneau spécial placé dans l'angle sud-ouest du caldarium; près du caldarium, un fourneau chauffait cette salle tiède (le tepidarium).

(2) Mau, Pompéii, p. 180-182-188.

(3) L. Drappier, Les thermes de Thuburbo majus, dans Bulletin archéologique du Comité..., 1920, p. 58 et p. 68 .

(4) L. Drappier, Les thermes d'El Djem, ibid., 1920, p. 466.

(5) Rapports de M. A. Merlin à la Commission de l'Afrique du Nord, ibid., 1917, p. cxill : de MM. Poinssot et Lantier, ibid., 1923, p. cxlix.

(6) Ibid., 1916, p. 179 ; 1917, p. 266. 
Cuicul, de même en Algérie, outre l'eau fournie par un aqueduc important, les grands thermes recevaient de deux réservoirs l'eau de pluie qui s'y trouvait emmagasinée (1). A Vertillum (Vertault), non loin d'Alésia, après avoir décrit la canalisation qui servait à l'alimentation des thermes, F. Daguin, dans son étude sur les fouilles de Vertault, indique que "le conduit aboutissait... à un réservoir servant à emmagasiner les eaux. Ce réservoir, de forme irrégulière, est creusé dans la roche et les parois ne présentent aucune trace de revêtement en maçonnerie... Il est probable que le réservoir était alimenté par les eaux d'une source captée à une certaine distance (2). Dans tous les cas les eaux de pluie venaient aussi augmenter à l'occasion la provision nécessaire aux thermes ; car on a reconnu sur un point du parcours de la conduite une ouverture pratiquée dans le dallage supérieur, qui vraisemblablement permettait à l'égout des toits voisins de se déverser dans le canal d'amenée " (3).

Or, en 1909, Victor Pernet a découvert, à peu de distance au nord des substructions que nous avons déblayées cette année, une pièce, dans laquelle il voit un citerneau et qu'il décrit en ces termes : « ... une pièce de forme rectangulaire allongée du nord au sud et mesurant 4 mètres sur $2 \mathrm{~m}$. 80 . Les murs de cette pièce sont bien construits en petit appareil avec joints passés au fer ; par endroits ils sont presque intacts sur une hauteur de près de 3 mètres. Les quatre murs qui entourent la pièce ne présentent aucune ouverture; à l'extérieur, ils sont entourés de partout par le roc naturel ; l'espace qui restait vide entre eux et le roc avait été rempli par de l'argile malaxée et battue. Le sol de la pièce était formé de haut en bas par : $1{ }^{\circ}$ Une couche de sable de rivière; $2^{\circ}$ un hérisson de $0 \mathrm{~m} .15$ d'épaisseur; $3^{\circ}$ une couche d'argile malaxée; $4^{\circ}$ le roc lui-même. " De tous ces détails il résulte que cette pièce n'était autre chose qu'un citerneau, au sol et aux parois duquel on avait assuré la plus grande étanchéité possible. "Au sud-est de ce citerneau, fait observer V. Pernet dans le Journal des Fouilles, on a découvert en 1908 une rigole en pierre qui longeait uns construction. Il est possible que cette rigole aboutit au citerneau. L'eau qu'elle y amenait provenait-elle des toitures ou des cours et rues avoisinantes ? Il est à remarquer qu'on n'a trouvé autour du citerneau aucun débris de tuile de couverture (4). »

Toute une partie de l'édifice à double colonnade se trouve précisément au sud-est de ce citerneau. Il ne serait pas impossible que la conduite d'amenée,

(1) Ibid., 1930-1931, p. 93

(2) Ce n'est là qu'une hypothèse de F. Daguin; rien dans la description du réservoir ou des ruines immédiatement voisines ne parait se rapporter à un aqueduc ; ce réservoir donne plutôt l'impression d'une citerne.

(3) F. Daguin, Les fouilles de Vertault, dans Mémoires de la Société des Antiquaires de France, 1896, p. 349-350.

(4) Bulletin de la Société des Sciences de Semur, t. XXXVII, ann. 1910-1911, p. 91. 
dont la rigole de pierre serait un fragment, ait abouti à notre pièce $n^{0}$ VII, large seulement de $0 \mathrm{~m} .70$; cette étroitesse est tout à fait anormale et ne peut s'expliquer, semble-t-il, que par la présence d'une conduite entre ses deux murs.

Rien donc, dans les découvertes que nous avons faites cette année, ne s'oppose à notre interprétation de cet édifice à double colonnade. Mais il ne faut pas, pour en comprendre la disposition, se rapporter aux grands balnéaires aujourd'hui connus en Italie ou dans les diverses provinces de l'Empire romain ; il convient de ne pas oublier, comme nous l'avons indiqué plus haut, qu'Alésia était simplement le chef-lieu d'un pagus de la Civitas Aeduorum. Ses thermes se composaient d'une série de chambres parallèles s'ouvrant sur une galerie ou un portique, qui servait ou de promenoir ou de palestre. Si la façade avait reçu un aspect monumental, la décoration intérieure était plus modeste; les procédés de chauffage étaient d'une grande simplicité ; les deux puisards, que nous avons signalés, fournissaient peut-être un mode, singulièrement imparfait, d'évacuation des eaux. Nous ne voyons pas quelle autre destination pourrait être assignée à cet édifice certainement public en raison de son importance, de son caractère monumsntal et di la présence de l'hypocauste.

$\mathrm{Si}$ nous avions recueilli dans ses ruines des objets mobiliers en nombre. ces trouvailles auraient pu nous fournir des indices peut-être significatifs. Mais la moisson de tels objets a été à peu près nulle, abstraction faite des poteries et fragments de poteries que nous avons retirés du puisard de la pièce V. Déjà "n 1908 V. Pernet avait été frappé de ce fait et voici comment il l'expliquait : "Le sol des chambres n'est. par endroits, qu'à $0 \mathrm{~m}$. 05 de la surface du sol actuel... La culture et le temps ont fait leur œuvre de nivellement... Aucune trouvaille importante n'est faite dans ce dédale de murs de fondations... Tout ce qui se rapportait à l'édifice lui-même (sculptures, enduits intérieurs, objets perdus) a disparu, parce que depuis des siècles on cultive le Mont Auxois et que la charrue ne cesse de tourner et de retourner cette mince couche de terre de $0 \mathrm{~m} .05$ à $0 \mathrm{~m} .10$ d'épaisseur qui recouvre le pavage du rez-de-chaussée (1). "

Outre les matériaux de construction et quelques éléments d'architecture que nous avons mentionnés plus haut, seules. en dehors des poteries du puisard de la pièce $n^{0} \mathrm{~V}$, une hache polie en jadéite et de rares monnaies ont été trouvées.

\section{II. - L'habitation située à l'est de l'édifice à double colonnade.}

A l'est de l'édifice à double colonnade, nous avons dégagé une construction composée de deux pièces rectangulaires, séparée de la salle III, que nous considérons comme une annexe des thermes, par un espace libre, sorte de couloir

(1) Bulletin de la Société des Sciences de Semur, t. XXXVI, ann. 1908-1909, p. 392. 
large de $0 \mathrm{~m} .75$ (fig. 1). Nous avons eu souvent l'occasion de remarquer que de telles séparations existaient à Alésia entre deux habitations voisines; la mitoyenneté ne parait pas y avoir été de pratique courante. A l'est, cette habitation donnait sur une petite rue qui descendait vers le nord, en longeant à l'ouest le sanctuaire d'Ucuetis et Bergusia ou Monument à crypte, et qui est dénommée dans le rapport de 1908 Voie Traversière (1).

Dans le rapport provisoire rédigé au mois de septembre dernier, nous avons décrit les deux pièces rectangulaires mises au jour au début de la campagne de cette année. Nous nous permettons de rappeler cette description en la complétant et en la précisant quelque peu.

De la plus orientale des deux pièces, $\mathrm{n}^{0}$ I du plan, seuls sont bien conservés les murs de l'ouest et du nord, tous deux en retrait plus ou moins régulier sur un mur plus ancien. Le mur de l'est est tout à fait grossier; de celui du sud de rares vestiges subsistent seuls. Au fond de cette pièce, le sol naturel a été percé en trois points ; les excavations ainsi creusées, de formes irrégulières, remplies de terre meuble, ressemblent à celles que nous avons déjà rencontrées en d'autres points du Mont Auxois et que nous avons signalées dans notre rapport sur les fouilles de 1937 : "Excavations, disions-nous alors, irrégulières, parfois très rapprochées les unes des autres, de dimensions inégales et que nous avons trouvées remplies de terres de déblai. Les avait-on ménagées à l'origine pour donner plus de stabilité aux amphores pointues ou aux grandes jarres à base convexe? Nous ne pouvons à cet égard formuler que des hypothèses. "Une solution certaine de ce problème archéologique n'a pas été trouvée.

La seconde pièce, $n^{0}$ II du plan, située à l'ouest de la précédente, semble de construction plus soignée, bien que le mur du sud ait complètement disparu. Le mur qui la sépare de la pièce $\mathrm{n}^{0}$ I a gardé, dans sa partie supérieure, plusieurs assises de moellons réguliers, entre lesquelles on voit encore en place du mortier de chaux de couleur blanche. Au-dessous de ces assises, le mur qui correspond sans doute au niveau de la cave gallo-romaine, est encore fait de moellons à peu près bien appareillés et descend jusqu'au niveau du sol naturel. Plus bas, au-dessous de ce niveau, un mur, fait de pierres plates disposées en assises régulières, révèle l'existence, à 3 mètres de profondeur environ, des vestiges d'une construction plus ancienne. Trois niveaux se distinguent en ce point fort nettement : 10 Un rez-de-chaussée ; - $2^{0}$ Une cave gallo-romaine probablement du ${ }_{11}{ }^{e}$ et $d u$ III ${ }^{\mathrm{e}}$ siècle après J.-C. ; - $3^{\circ}$ Plus bas, une habitation d'aspect plus grossier, dont la partie souterraine avait entamé le sol naturel et atteint la strate rocheuse déjà rencontrée en d'autres points d'Alésia. Pour cette partie souterraine,

(1) Ibid., t. XXXVI, ann. 1908-1909, p. 358. 
l'emplacement d'une hutte gauloise avait peut-être été utilisée. Dès 1911, nous avons montré que les caves des maisons gallo-romaines d'Alésia ont souvent occupé les excavations ménagées par les Gaulois dans le sol naturel du Mont Auxois (1).

Cette pièce est directement contiguë à un hypocauste fouillé en 1908 (2), hypacauste construit sur l'emplacement d'une hutte gauloise, dont le pavement, situé au niveau du fourneau de l'époque gallo-romaine, était fait de terre glaise battue sans chaux. Immédiatement au nord de cet hypocauste se trouve la Cave dite à la Mater, parce qu'on y a découvert la statuette de la Déesse-Mère tenant des fruits entre ses genoux (3).

Les deux pièces que nous avons dégagées cette année, l'hypocauste et la Cave à la Mater, se succédant sans aucune séparation du sud au nord, appartenaient probablement à la même habitation, qui occupait, entre les Thermes et le Sanctuaire d'Ucuetis et Bergusia, l'angle formé par la Voie Traversière et la rue nouvelle mise au jour en 1942 .

III. - La rue nouvelle.

Cette rue, à peu près exactement dirigée d'est en ouest, est longue d'environ 15 mètres. Sa chaussée, large de 4 mètres, est faite d'un béton grossier composé de petits cailloux très usés. Elle aboutit au mur est des Thermes, en un point où avait été ménagée une baie ou porte latérale, dont le seuil se laisse encore reconnaitre.

$\mathrm{Au}$ sud de la rue se voient les traces peu distinctes d'une construction, dont le pavement consistait en une mosaïque où se mêlaient de menues pierres et de petits morceaux de terre cuite, le tout noyé dans un mortier très solide (sur le plan $\mathrm{M}^{\mathrm{1}}$ ). Cette construction se trouvait au contact de la voie qui longeait la façade des Thermes et rejoignait non loin de là, le Forum de la ville.

Objets mobiliers.

Bien que la récolte d'objets mobiliers n'ait pas été, comme nous l'avons indiqué plus haut, très abondante, nous en avons pourtant recueilli quelques-uns dont l'intérêt nous paraît digne d'être signalé.

10 Pierre. - Outre les matériaux de construction et les éléments d'architecture déjà mentionnés, nous avons trouvé une hache polie en jadéite et un poids gallo-romain avec inscription.

a) La hache en jadéite, de nuance verte, est dans un parfait état de conser-

(1) Bull. arch. Comité..., 1911, p. 324 et suiv.

(2) Bull. Soc. Sciences Semur, t. XXXVI, ann. 1908-1909, p. 361-363.

(3) Ibid., p. 374. 
vation (fig. 8). Longue de $0 \mathrm{~m} .08$, large au tranchant de $0 \mathrm{~m} .047$, sa plus grande épaisseur est de $0 \mathrm{~m}$. 018. Sa découverte pose un double problème, le problème de son origine, le problème de son usage.

Une discussion s'est élevée entre spécialistes de l'archéologie préhistorique sur le lieu de provenance du jade et de la jadéite. Pour les uns, ces pierres étaient d'origine orientale, asiatique; mais cette théorie est aujourd'hui abandonnée, depuis que l'on a constaté la présence de jade et de jadéite à l'état brut, d'une part dans la région alpestre en Suisse et en Styrie, d'autre part en Bretagne dans le Morbihan et au nord de la Ven-

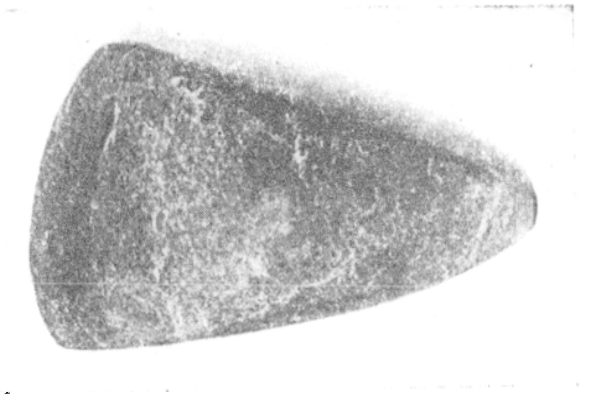

Fis. 8. Hache en jadéite polie. dée dans le pays de Retz ou Rais (1). Déchelette pense que dès les temps néolithiques le commerce a pu disperser dans un rayon très étendu les haches faites de ces substances minérales.

Mais comment expliquer la présence d'une hache de pierre polie dans une ronstruction de l'époque gallo-romaine, en un point où n'existe aucun gisement néolithique. (ie n'est pas la première fois que le fait est constaté. Même si l'on

fait abstraction des cas où de telles haches étaient ccrtainement votives (2), on peut citer de nombreuses découvertes de haches de pierre polie dans des ruines de maisons ou dans des sépultures gallo-romaines $(3)$. Ces haches passaient pour être des talismans contre la foudre; cette superstition a survécu, non seulement pendant les premiers siècles du Moyen-Age, sous les Mérovingiens et les Carolingiens, mais même dans les temps modernes (4). Il est probable que notre hache de jadéite polie était placée quelque part dans les substructions que nous avons déblayées comme un fétiche protecteur.

b) Le poids de pierre est une masse de forme cylindrique, d'un diamètre de $0 \mathrm{~m}$. 25, d'une hauteur de $0 \mathrm{~m} .16$ (fig. 9 et 10 ).

L'anneau de métal qui servait à la manier a disparu. Deux inscriptions ont été gravées sur ce poids d'une part LXX, d'autre part non loin de la première, $\mathrm{L}^{\mathbf{v}}$; dans ce second texte le $\mathrm{V}$ est beaucoup plus petit que le $\mathrm{L}$. Pourquoi ces deux marques dont l'une correspond au chiffre 70 , dont l'autre parait bien signifier 55 ?

(1) J. DÉchelette, Manuel d'archéologie préhistorique, t. I, p. 627-628; A. Blanchet, Bull. Soc. Antiquaires, 1896, p. 354-355.

(2) J. Formigé, ibid., 1925, p. 129 et suiv.; cf. L. DE VESL', Les fana ou petits temples gallo-romains de la région normande, passim; J. ToutaIs, Les Culles païens dans l'Empire romain, t. III, p. 366.

(3) J. Formigt, loc. laud.; Ad. Blanchet, ibid., p. 133.

(4) P. SÉbillot, Le Folklore de France, t. IV, p. 67. 
Dans son état actuel, le poids pèse $16 \mathrm{~kg} .500$; mais il est démuni de son anneau et du scellement, sans doute au plomb, qui rattachait l'anneau à la masse de pierre. Anneau et scellement, qui devaient être assez puissants pour porter plus de 16 kilos, représentent bien 1 kilo ou $1 \mathrm{~kg} .1 / 2$, ce qui porte le poids total de l'objet à 18 kilos approximativement. Or 55 livres romaines de 327 gr. 45 pèseraient exactement $1.800 \mathrm{gr}$. 75. C'est donc le chiffre $L^{v}$ qui donne le poids véritable d'après le système des poids et mesures romain. Mais alors que signifie l'autre marque LXX que porte l'objet?

Ici plusieurs hypothèses peuvent être envisagóes.
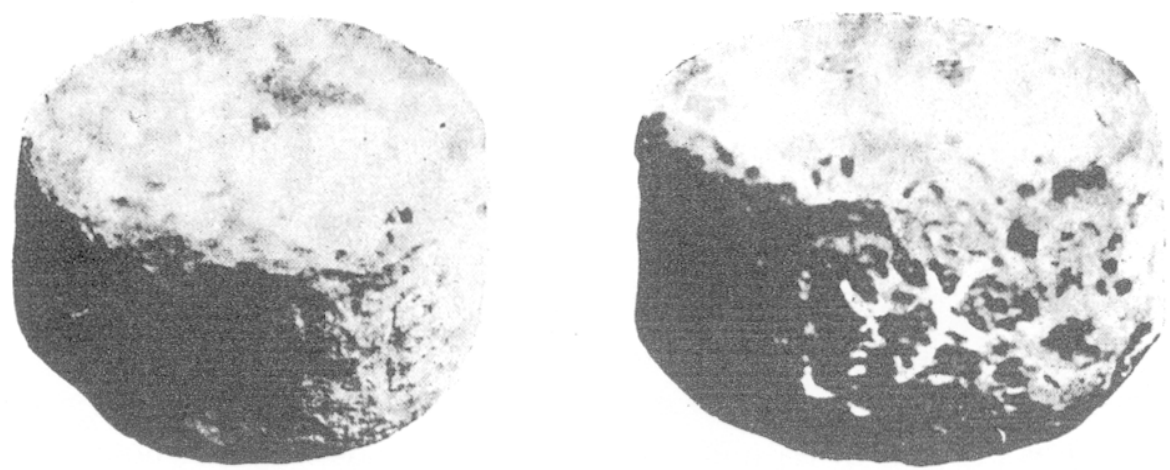

FIG. 9 et 10. Le poids de pierre.

10 La marque LXX proviendrait du défaut d'exactitude qui a été maintes lois constaté en matière de métrologie antique et que signalent Bœckh et Hultsch (1). Un exemple significatif d'erreur de ce genre est cité par J. Marquardt: un poids portant la marque $\mathrm{X}$, c'est-à-dire inscrit comme pesant 10 livres avec l'estampille officielle de L. Junius Lucilianus, légat impérial de la legio I Italica, pèse en réalité $5.558 \mathrm{gr}$. 05 , soit à peu près exactement 17 livres (2);

$2^{\circ}$ On peut supposer que nous avons affaire ici à un faux poids. Nous savons que l'usage en était répandu dans l'Empire. Au Digeste se trouve une mention des pondera iniqua par Ulpien (3). Il y est fait allusion dans quelques inscriptions, en particulier à Tusculum (4) et près d'Ariminum (5). L'erreur volontaire consistait tantôt dans l'inscription d'un poids inférieur, tantôt dans l'inscription p. 156.

(1) В๔скн, Metrologische Untersuchungen, p. 188; HuLtsch, Griechische und romische Metrologie,

(2) L'organisation financière chez les Romains, trad. fr. A. VıgIÉ, p. 93, n. 5.

(3) L: XIX, tit. 1, Ir. 32.

(4) C. I. Lat., XIV, 2625.

(5) Id., $\mathrm{XI}, 6375$. 
d'un poids supérieur au poids réel (1). Dans le cas de notre poids d'Alésia, le vendeur aurait essayé de faire payer à l'acheteur 70 livres romaines au lieu de 55 livres, poids réel de la denrée qu'il lui livrait.

A ces deux premic̀res hypothèses une objection sérieuse peut être opposée. Dans l'un et l'autre cas, l'inscription du chiffre $L^{v}$ nous incite à penser qu'il y aurait eu vérification et par suite correction du poids annoncé par la marque LXX. Que de telles vérifications eussent lieu, nous le savons certainement. La présence de poids étalons dans les villes est attestée par plusieurs documents (2). Ces poids étaient conservés dans un bâtiment spécial, le Ponderarium, et c'étaient les édiles municipaux qui étaient chargés de ce service (3).

Mais, si notre poids fut soumis à la vérification et si la marque exacte $L^{v}$ y fut inscrite, on se demande pourquoi la marque LXX y est restée gravée, sans qu'aucun essai de la gratter ou de la marteler soit apparent sur la pierre.

Une troisième hypothèse nous parait plus acceptable ;

$3^{0}$ Les érudits qui ont spécialement étudié la métrologie romaine ou qui ont eu l'occasion de s'en occuper, frappés des anomalies que nous venons de mentionner, sont entrés pour les expliquer dans une autre voie. Ils se sont demandé si les chiffres inscrits sur les poids ne correspondaient pas à d'autres unités que la livre romaine de $327 \mathrm{gr}$. 45 , soit à des unités plus anciennes encore usitées parfois (4), soit à des unités locales ou régionales (5). Et, pour ce qui est de la Gaule romaine, Camille Jullian n'est pas éloigné de croire à la survivance d'unités gauloises : après avoir reconnu que " le système romain des poids et mesures fut imposé à la Gaule dès le lendemain de la conquête ", il ajoute "mais je doute d'une proscription absolue des systèmes indigènes... On dut tolérer différentes mesures du pays et quelques-unes même, comme la lieue, parurent plus tard si commodes que l'État finit par les préférer dans certains cas aux siennes propres" $(6)$.

Dès lors ne peut-on pas expliquer la double inscription de notre poids par le maintien d'un système celtique auprès de la livre romaine. Le poids aurait pesé d'une part 55 livres romaines, d'autre part 70 unités de poids indigènes.

(1) Hultsch, op. laud., p. 156 : zwar finden sich nicht nur Stäcke mit bedeutendem Mindergewicht, sondern auch solche mit merklichem Uebergewicht.

(2) Ét. Michon, Dictionnaire des antiquités..., de Daremberg et SAglio, s. v. Ponderarium.

(3) C. I. Lat., XI, 6375 ; XIV, 2625.

(4) Вескк, op. laud., p. 188-189 : ... die beglaubigten Gewichte, die doch schwerlich betrugerisch waren, zeigen grosse Unterschiede. Liegt hiervon der Grund etwa darin, dass in andern Zeiten anderes Gewicht in Rom und Italien war?

(5) Hultsch, op. laud,. p. 156 : après avoir cité les poids qui s'écartent du système de la livre romaine, ... einem Theile derselben liegen abweichende stădtische und provinziale Pfunde zu Grunde... en note à ce passage, Hultsch déclarait qu'une étude des poids provinciaux rendrait grandement service, serait verdienstlich.

(6) Histoire de la Gaule, 1. 1, p. 345-346. 
Si l'on admet qu'intact et complet ce poids pesait 18 kilos, on obtiendrait pour l'unité pondérale gauloise un chiffre $18.000: 70=257$. gr. 14 . Sans doutc ce n'est là qu'une hypothèse, mais d'une part elle nous paraît pouvoir expliquer la présence des deux inscriptions également intactes gravées sur le poids; d'autre part elle n'est pas en contradiction avec ce que nous savons de l'attitude adoptée par l'Administration romainc à l'égard des poids et mesures indigènes dans les diverses provinces de l'Empire.

$2^{0}$ Bronze. - Dans les terres qui remplissaient l'habitation située à l'est de

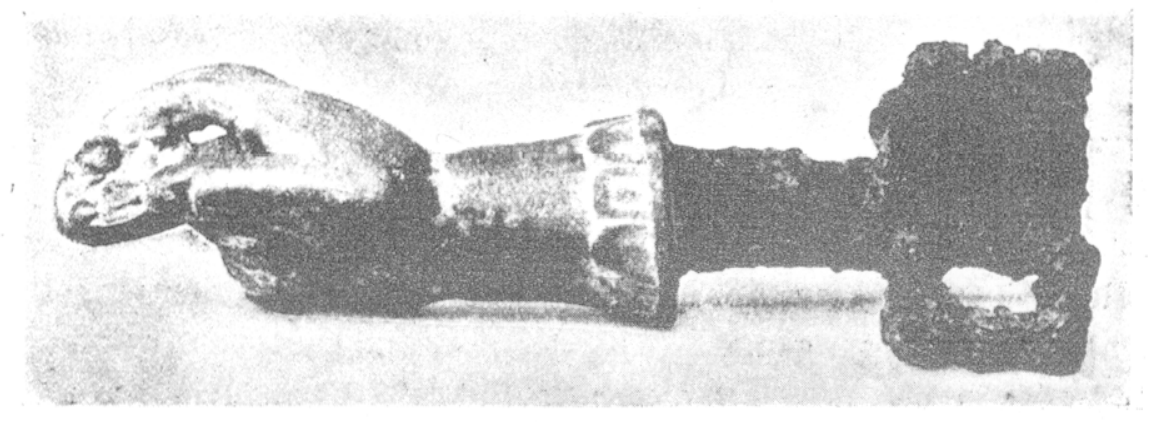

FIG. 11. Clef avec poignèe de bronze en forme de main.

l'édifice à double colonnades, nous avons recueilli deux clefs de bronze d'un intérêt exceptionnel.

a) Une clef (fig. 11) dont le panneton et la partie de la tige voisine du panneton sont en fer. Le panneton est à trois pans, dont l'un est perpendiculaire au plan des deux autres. Ces deux derniers sont évidés ; l'autre est formé de trois dents très rapprochées l'une de l'autre. La tige est pleine. La partie la plus curieuse, la plus originale de l'objet est la poignée de bronze. Elle représente une main féminine, très bien modelée, d'une grâce très fine. Cette main tient entre le pouce et l'index allongés une patère dans laquelle on distingue des fruits; les trois autres doigts sont repliés. Tous les détails du geste sont indiqués avec une exactitude et un naturel très remarquables. Le poignet qui précède la main parait sortir d'une manche qui se termine par une bande de godrons ou festons allongés en forme de lambrequins; c'est là un ornement assez rare dans les costumes féminins de l'antiquité romaine. Longueur totale de la clef : $0 \mathrm{~m} .12$; longueur de la poignéc : $0 \mathrm{~m}$. 07 . On connait de nombreuses clefs antiques à poignée de bronze sculptée. Les motifs les plus fréquents semblent avoir été soit un avant-corps d'animal, surtout de lion (1), soit une tête d'animal, lion

(1) Babelon et Blanchet, Catalogue des bronzes antiques de la Bibliothèque nationale, no 1887 ; F. LIGER, La ferronnerie ancienne et moderne, t. I, pl. 8 ; S. REINACH, Bronzes figurés de la Gaule romaine, nos 446-450-454-455 bis. 
panthère, mulet, chien (1). Une seule clef, à notre connaissance, possède une poignée analogue à la nôtre : c'est le no 1895 du Catalogue de Babelon ct Blanchet, dont la poignée a la forme d'une main "qui tient une boule de fard "; mais elle est moins fine et d'un art moins achevé.

Devons-nous essayer de découvrir les raisons pour lesquelles ce type de poignée, qui est loin d'être banal, a été choisi par son possesseur ? Nous avons été frappé d'un rapprochement, peut-être purement accidentel, pourtant suggestif. Les deux pièces que nous avons fouillées à fond cette année à l'extrémité orientale de notre chantier, appartiennent, comme nous l'avons indiqué plus haut, à une habitation dont les vestiges se prolongent au nord et dont faisait partie la cave dite à la Mater. On sait que l'imagé de la Déesse-Mère trouvée dans cette cave, se distingue par le groupe de fruits de grosseurs diverses, les uns arrondis, les autres ovales ou pointus, que la Déesse tient dans son giron sur une étoffe qui s'enfonce légèrement entre ses genoux (2). "Ces fruits, remarque Ad.-J. Reinach, sont les fruits ordinaires de nos jardins ; les dimensions différentes indiquent peut-être des pommes, des prunes, des noix, peut-être aussi les produits du potager à côté de ceux du verger. "Or c'est exactement le même aspect que présentent les fruits réunis sur la patère que tient notre main de bronze, fruits de grosseurs et de formes différentes. Serait-ce une conclusion téméraire de supposer que la statuette de la Déesse-Mère et la clef de bronze, trouvées à quelques mètres l'une de l'autre, presque certainement dans la même habitation, attestent chez les maitres de cet immeuble un intérêt particulier pour les fruits et nous les révèlent comme les propriétaires d'un riche verger dans la campagne d'Alésia ? La rareté du motif choisi pour orner la poignée de la clef et de même la rareté du type de la Déesse-Mère nous paraissent justifier et, nous l'espérons, excuser cette hypothèse.

b) Une seconde clef, toute de bronze, dont la poignée et le panneton sont également ouvragés (fig. 12). Le panneton se compose de deux pans en équerre :l'un, perpendiculaire à l'axe général de la clef, cst formé de trois dents pleines séparées, longues de $0 \mathrm{~m} .02$; l'autre, qui prolonge le plan de la poignée est un carré de $0 \mathrm{~m} .02$ de côté, percé de découpures assez compliquées ; au-dessous de ce carré un petit bouton surmonté d'une gorge contribue, avec les détails précédents, à nous renseigner sur la structure délicate de la serrure que cette clef permettait d'ouvrir. Quant à la poignée, elle a la forme d'un arc, qui se recourbe en deux petites volutes, le tout s'appuyant sur une sorte d'embase étroite et allongée ; entre cette embase et le panneton se trouve un ornement formé, lui aussi, de deux petites volutes divergentes. Des clés de ce type, à panneton en équerre

(I) S. Reinach, op. laud., nos 451-453-456-459.

(2) Ad.-J. Reinach, La nouvelle Déesse-Mère d'Alésia, Pro Alesia, 30 annèe, p. 425 et suiv. 
et fort ouvragées, ne sont pas rares (1). Notre exemplaire se distingue par son parfait état de conservation et l'harmonieuse proportion de ses diverses parties.

Nous devons encore signaler comme trouvailles : un ornement en métal (bronze?) argenté, ayant sans doute fait

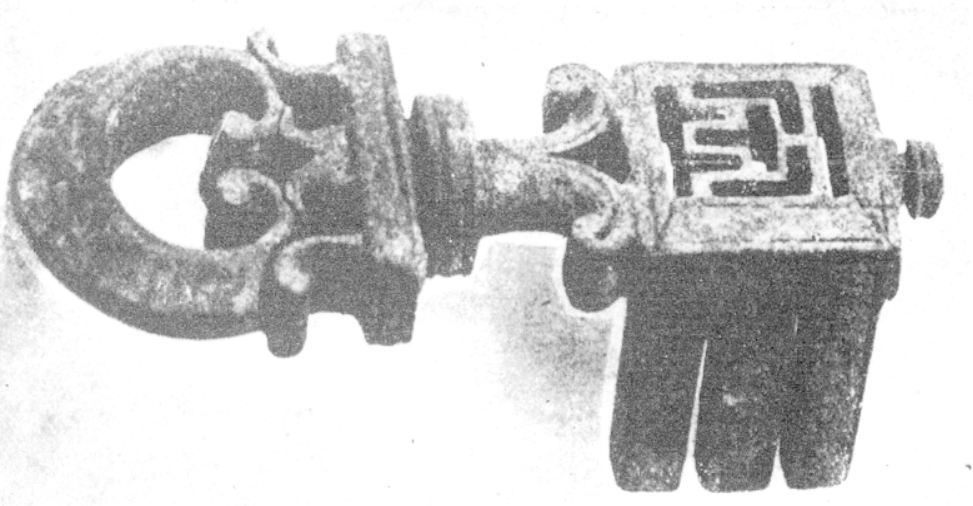

Frg. 12. Clef de bronze à panneton en équerre. partie d'un harnais de cheval, qui confirme une fois de plus le texte souvent cité de Pline l'Ancien, Nat. Hist., XXXIV, 48, 162 : deinde et argentum incoquere simili modo coepere equorum maxime ornamentis et jugorum in Alesia oppido ; - un

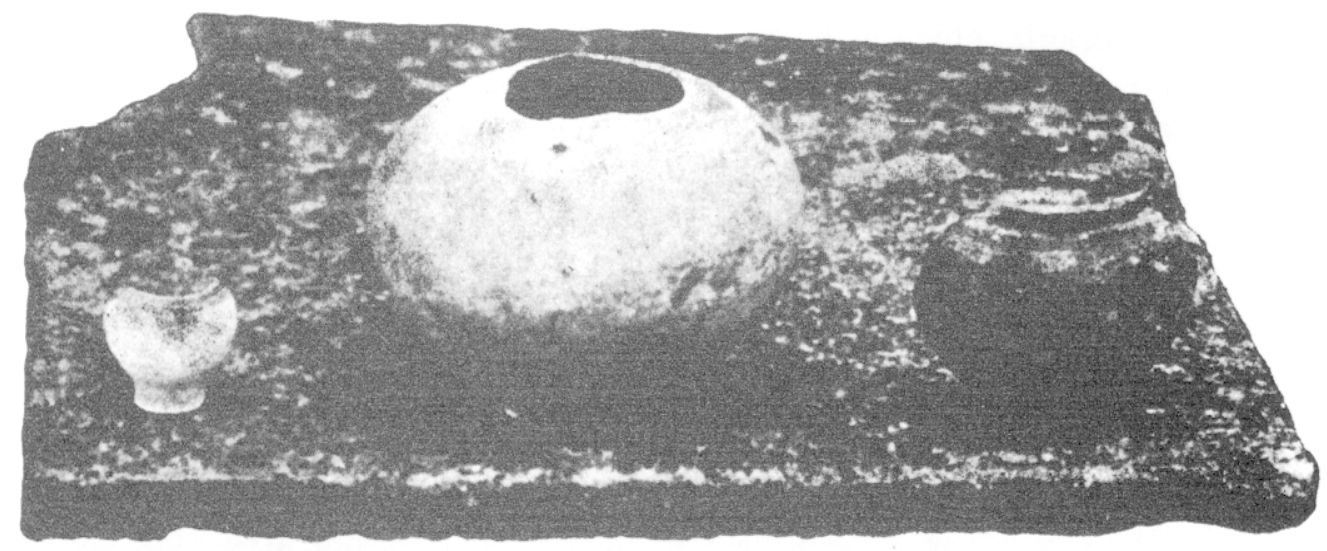

Fig. 13. Trois vases trouvés dans le puisard de la chambre $V$.

débris de petit miroir encore muni de deux rivets qui servaient à le fixer (diamètre : $0 \mathrm{~m}$. 065).

$3^{\text {o }}$ Terre cuite. - Les nombreux fragments de poteries que nous avons recueillis proviennent à peu près exclusivement du puisard découvert dans la

(1) Babelon et Blanchet, Calalogue..., nos 1888 et suiv.; Cagnat et Chapot, Manuel d'archéologie romaine, t. II, p. $422-423$, fig. $620^{2}$; cf. Guide to Greek and Roman life, p. 163. 
chambre $\mathrm{V}$ de l'établissement thermal. Ce sont, en très grande majorité, des morceaux de vases communs, amphores, cruches à une seule anse, plats ou coupelles, sans vernis ni décoration, en poterie rouge, grise ou noire.

Trois vases seulement ont été retrouvés presque intacts (fig. 13) : un vase minuscule ; haut de $0 \mathrm{~m}$. 042, à engobe blanc, sans doute un balsamaire ; - un vase en poterie légère, à panse très large, en forme d'ampulla, haut de $0 \mathrm{~m} .13$, dont le diamètre maximum mesure $0 \mathrm{~m} .17$, tandis que celui du fond n'est que de $0 \mathrm{~m} .09$; le col manque ; c'est sans doute une ampulla olearia; - un pot, de moindres dimensions, de forme analogue un peu moins accentuée, à large orifice, sans col ni anses.

En ce qui concerne la décoration, outre quelques débris de vases sigillés que nous décrirons plus loin, nous signalerons deux types particuliers : des lignes de stries tout autour de la panse; - un semis de points en relief, très fins sur un vase, un peu plus gros sur un autre, et donnant au toucher l'impression d'une râpe.

Bien qu'aucun vase sigillé n'ait été retrouvé intact, les fragments que nous avons recueillis méritent d'être mentionnés. Voici les principaux motifs qu'ils présentent (fig. 14) : au-dessous d'une, zone de godrons, panneaux successsifs de dimensions inégales, les uns plus larges contenaient des personnages peu distincts, les plus étroits occupés par des ornements floraux plus ou moins stylisés ; animaux courants, chien et lièvre (?) ; entre les groupes ornements en forme de fers de lances ou d'arbres stylisés (sapins ?), encadrés de lignes traitées à la manière de cordages ou de cordonnets ; - fragment de coupelle unie, portant au fond la marque BASSVS; - deux fragments d'un plat avec la marque OF. MARTIAL; - un autre fond de plat où je crois lire la signature VOLVS.

La poterie vernissée rouge est encore représentée dans nos trouvailles par un certain nombre de fragments unis, sans décor ni sigles.

En dehors de ce puisard, nous avons trouvé dans les déblais provenant des chambres VII, VIII et IX, un de ces instruments d'éclairage comparables à nos bougeoirs modernes et désignés, dans le Manuel d'archéologie romaine de R. Cagnat et V. Chapot, sous le nom de chandeliers portatifs (1); c'est une patère creuse, mesurant $0 \mathrm{~m} .075$ de diamètre, profonde de $0 \mathrm{~m} .026$, portant en son centre une petite cavité cylindrique destinée à recevoir une chandelle, candela, faite de suif, ou une bougie, cereus, faite de cire. Plusieurs exemplaires du même tỵpe de luminaire ont été déjà trouvés à Alise et figurent au Musée d'Alésia ;

40 Verre. - Nous avons trouvé un morceau d'un vase en verre bleu, côtelé,

(1) T. II, p. 466 , fig. 660 . 
orné, en travers des côtes, de dessins blancs, à l'aide desquels le verrier semble avoir voulu figurer des sortes d'attaches destinées à maintenir les côtes bien appliquées sur le corps du vase :

$5^{0}$ Monnaies. - Nous avons trouvé au cours de nos travaux 4 monnaies gauloises et 9 monnaies romaines.
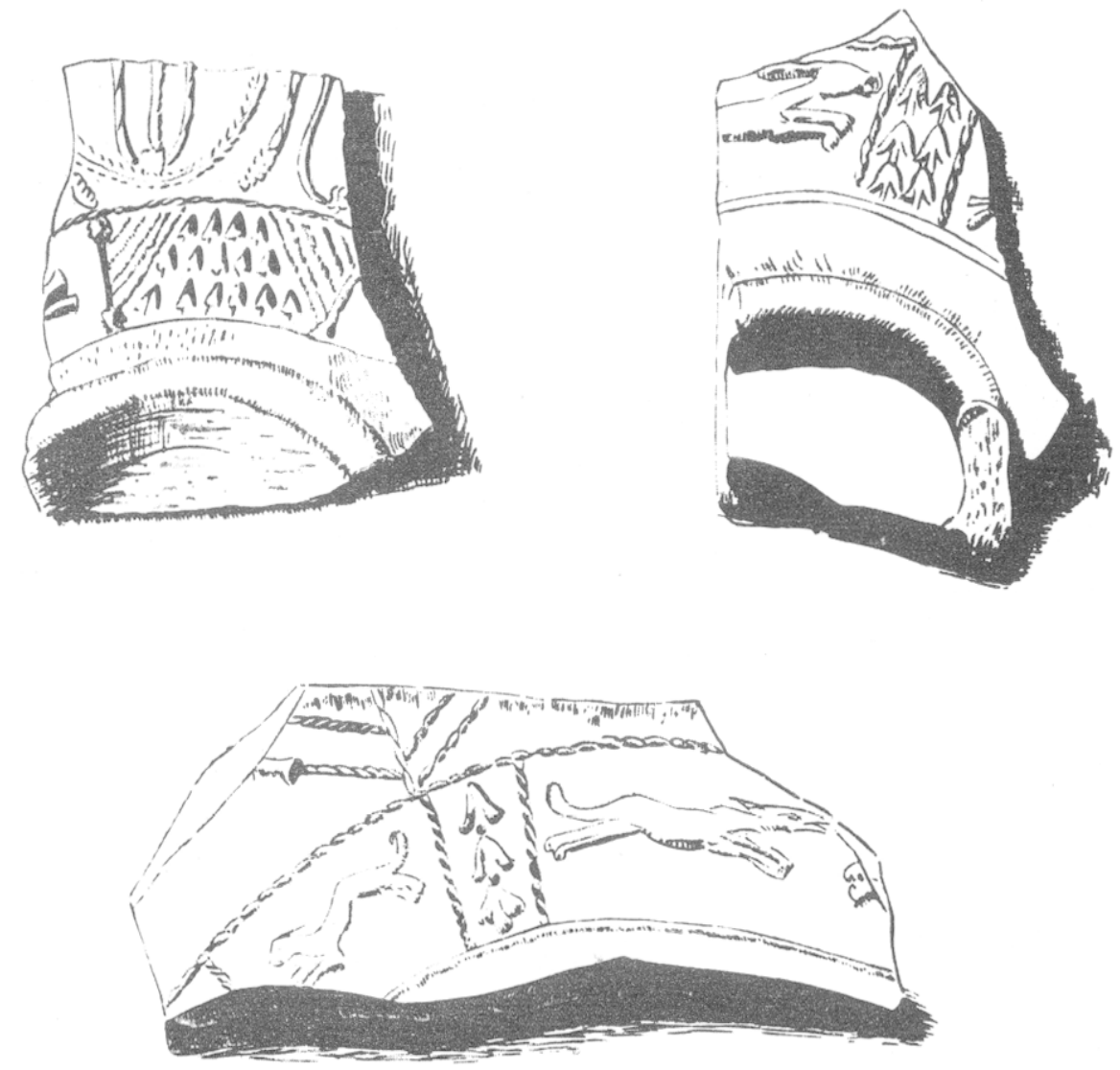

FIG. 14. Fragments de vases sigillés. (Dessins de Mme J. T.)

Des 4 monnaies gauloises, il y en a 3 qui paraissent appartenir au monnayage de la tribu des Leuci (Vosges et Haute-Marne), en raison de l'image de sanglier à gauche plus ou moins dégénérée qu'elles présentent au revers. La $4^{\mathbf{e}}$ est pcu distincte.

Les monnaies romaines se divisent en deux groupes : les unes sont du $\mathrm{I}^{\mathrm{er}}$ siècle de l'Empire, les autres du milieu du in ${ }^{\mathrm{e}}$.

1. Petit bronze. Tête casquée à droite. Rv. Taureau marchant à gauche. [Ge]RMANVS [I]NDVTILLIL. Sur cette monnaie très répandue en Gaule, v. Ad. Blanchet, Traité des monnaies gauloises. p. 253. 
2. Moyen bronze. Envers peu distinct : une tête à droite (Auguste ou Tibère ?). Rv. L'autel de Lyon. ROM ET $A V G$.

3. Moyen bronze. Tête de Néron à gauche. [Imp. Nero Cae]SAR AV $[\mathrm{g}]$ $P M[\ldots . . .$.$] . Rv. Indistinct; on aperçoit seulement le \mathrm{G}$ de la formule bien connue SC.

4. Moyen bronze. Tête de Vespasien à droite. IMP. CAES. VESPASIANVS..... Rv. La Foi debout à gauche tenant une patère de la main droite et une corne d'abondance dans le bras gauche. FIDES...SC. Cf. CoHEN, 1 re édit., t. I, p. 302, no 279-281.

5. Moyen bronze. Tête de Maximin lauré à droite. IMP. MAXIMINUS PIVS $A V G$. Rv. Victoire ailée marchant rapidement à droite, tenant une couronne de la main droite et une palme dans son bras gauche. VICTORIA AVG. Dans le champ, $S C$. - Cf. Cohen, $1^{\text {re }}$ édit., t. IV, p. 98, n $^{\text {os }} 90-91$; Mattingly et Sydenham, t. IV, 2, p. 145, nos 67-68-69. - Date de l'émission, entre mars 235 et janvier 236.

6. Petit bronz.e. Tête de Valérien radié à droite. IMP. C. P. LIC. $V A L E R I A N V S A V G$. Rv. Victoire à gauche tenant une couronne de la main droite et une palme de la main gauche. VICTORIA AVGG. Cf. CohEN, 1 re édit.; t. IV, p. 329, no 142 ; - Mattingly et Sydenham, t. V, 1, p. 48, nos 124-126? Monnaie émise probablement en 254 ou 255. L'absence des mots Pius et Felix dans la titulature de l'empereur indique une date antérieure à 255.

7. Petit bronze. Tête de Victoire radié à droite. IMP. C. VIC $[t]$ ORINVS $P$. [F. Aug.]. Rv. La Piété debout à gauche faisant une libation sur un autel

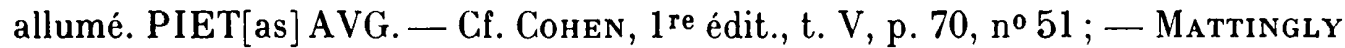
et Sydenham, t. V, 2, p. 392, no 57 et pl. XIV, 9 ; - Date de l'émission, 265-267.

8. Petit bronze. - Tête de Claude II radié à droite. - IMP. C. CL[audiu]S $A V G$. - Rv. L'Abondance debout à gauche, tenant des épis de la main droite, une corne d'abondance dans la main gauche. ANNONA AVG. -

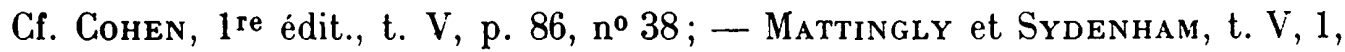
p. 213, no 18, p. 222, no 139.

9. Petit bronze. Tête d'empereur radié à droite. - IMP. C. TE....VS. P. F. AVG. - Rv. Personnage féminin debout à gauche [.......] TAS AVGG. Est-ce une monnaie de Tetricus ? La titulature est trop indécise, bien que les deux lettres $T E$ paraissent certaines : il y a un espace trop large entre ces deux lettres et la fin du mot. De même au revers le nom de la déesse est trop mutilé pour qu'on essaie de le reconstituer.

Si l'on ajoute aux monnaies ci-dessus, recueillies cette année, celles qui ont été découvertes en 1908-1909. lars des fouilles superficielles de l'édifice à double colonnade : soit une moitié d'un bronze de la Colonia Nemausus aụ type du 
crocodile, un bronze de Tibère à l'Autel de Lyon, un moyen bronze de Néron, un grand bronze de Néron, un moyen bronze d'Hadrien, on établit une liste de pièces qui se divisent en deux groupes chronologiques assez nets : 1 er, du début de l'Empire presque vers le milieu du II $^{\mathrm{e}}$ siècle $; 2^{\mathrm{e}}$, les deuxième et troisième quarts du iII ${ }^{e}$ siècle. Une fois de plus cette liste fait apparaitre la lacune souvent
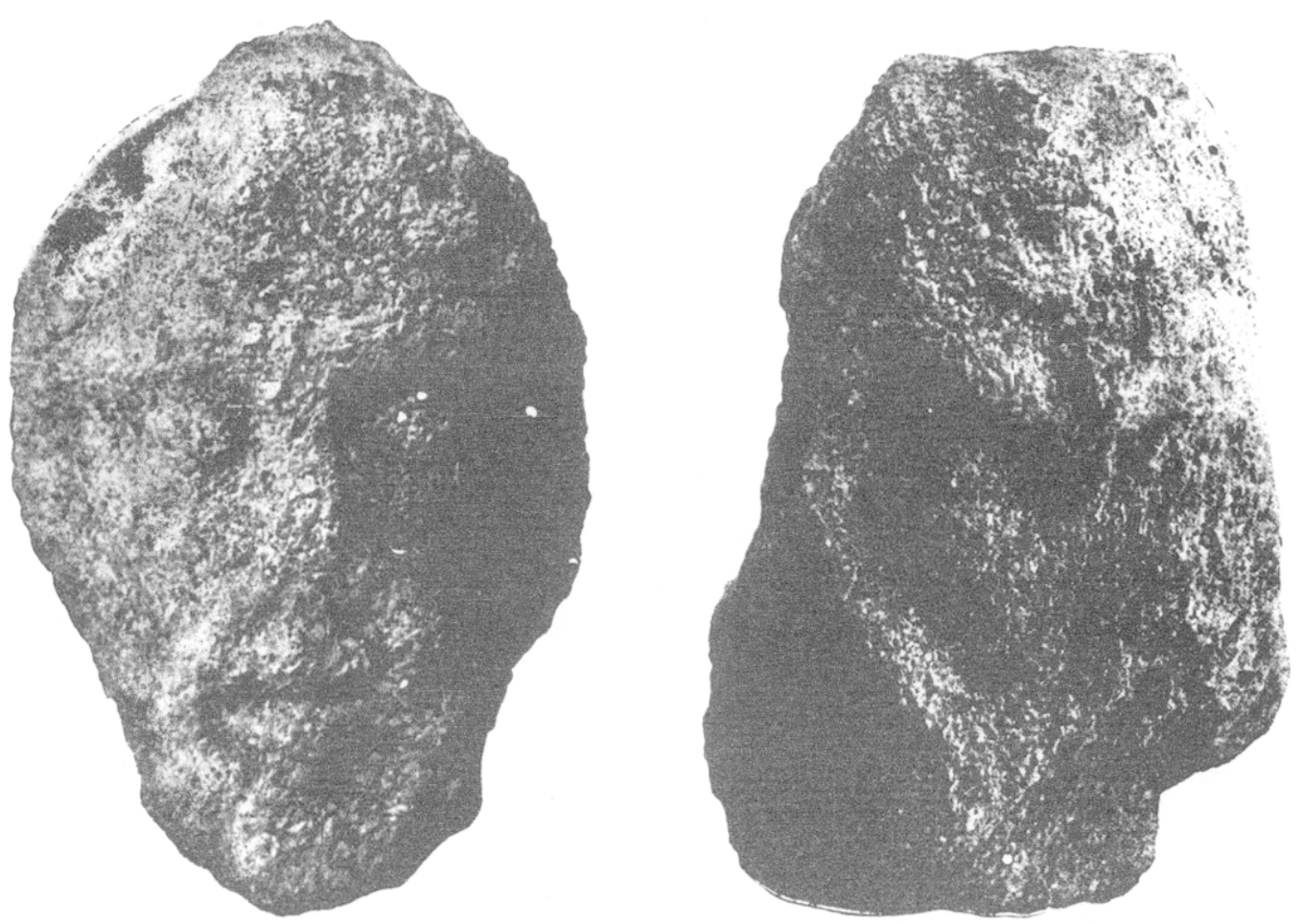

FIG. 15 et 16 . Tête barbue en pierre, à gros grain, lace et profil.

remarquée et signalée qui caractérise, dans la numismatique de la Gaule romaine, la période des Sévères.

Avant que n'ait été entreprise l'exploration méthodique de l'édifice à double colonnade, le surveillant des fouilles, R. Jacquenet, en procédant au nettoyage annuel de nos chantiers, a fait deux trouvailles intéressantes:

A) Dans un amas de déblais, situé le long du Chemin du Mont Auxois, près de l'emplacement d'une très belle cave découverte en 1913 (1), une tête en pierre d'un grain plutôt grossier et traitée grossièrement (fig. 15 et 16). Sauf le nez, elle est intacte au-dessus du cou, brisé par une section fort nette. Sa hauteur

(1) J. Toutain, Rapport général sur les fouilles exécutées à Alésia... en 1913, dans Bulletin archéologique du Comité, 1914, p. 388 et suiv. (ct. p. 366, fig. 2, cave F). 
cst de $0 \mathrm{~m}$. 11, sa plus grande largeur de $0 \mathrm{~m} .07$. Elle représente un personnage barbu, à la chevelure abondante, disposée en mèches épaisses, qui surmontent le front, encadrent la figure et par derrière tombent presque sur la nuque, où elles forment un bourrelet. Il ne semble pas que ce soit un portrait, mais plutôt une image de divinité. Aucun indice vraiment caractéristique ne permet de mettre sur ce visage un nom certain : pourtant la physionomie générale, l'aspect de la barbe qui est très courte et de la moustache tombante nous empêchent d'y voir l'un ou l'autre des grands dieux d'âge mur, Jupiter, Neptune ou Pluton. Ce pourrait être un Esculape ; mais nous sommes plus portés à y voir le dieu d'origine celtique, dont l'attribut principal est un maillet, qui fut assimilé, dans maintes régions de la Gaule, au Silvain italique et qui se retrouve, avec des physionomies variées, dans ces groupes de deux divinités assises côte à côte, si fréquents chez les Éduens. Comme la barbe et les cheveux sont indiqués largement, sans aucun usage du foret, comme d'autre part les pupilles ne sont pas creusées et que le globe de l'œil est représenté lisse, il est probable que ce petit morceau de sculpture remonte soit au $1^{\mathrm{er}}$ siècle de l'ère chrétienne, soit aux premières années du II ${ }^{e}(1)$. Quoi qu'il en soit, malgré son apparence plutôt fruste, cette œuvre de sculpture provinciale ne manque pas d'originalité;

B) Un curieux objet, composé d'une tige de bronze terminée à sès deux extrémités par deux boules du même métal, légèrement aplaties, entre lesquelles le long de la tige peuvent se déplacer deux rondelles, de bronze elles aussi. La tige de bronze mesure $0 \mathrm{~m} .075$ de long; les deux rondelles ont respectivement $0 \mathrm{~m} .036$ et $0 \mathrm{~m} .039$ de diamètre ; elles sont sobrement moulurées. Cet objet, dont la destination et l'usage précis nous échappent, servait peut-être à la décoration de quelque partie d'équipement, de harnachement ou de mobilier.

\section{J. Toutain,}

Président de la Société des Sciences de Semur, Directeur des fouilles d'Alésia.

(1) Ch. Dugas, dans le Dictionnaire des Antiquités, de Daremberg, Saglio et Pottia, s. v. Sculptura, p. 1149 : : Une façon nouvelle de représenter l'œeil en creusant la pupille apparatt... dans la sculpture en ronde bosse au temps d'Hadrien." 